\title{
Estimação de Parâmetros Genéticos de Medidas de Conformação, Produção de Leite e Idade ao Primeiro Parto em Vacas da Raça Gir Leiteiro ${ }^{1}$
}

\author{
Amauri Arias Wenceslau², Paulo Sávio Lopes ${ }^{3}$, Roberto Luiz Teodoro ${ }^{4}$, Rui da Silva Verneque ${ }^{4}$, \\ Ricardo Frederico Euclydes ${ }^{3}$, William José Ferreira ${ }^{2}$, Martinho de Almeida e Silva ${ }^{5}$
}

\begin{abstract}
RESUMO - Foram utilizados 573 registros zootécnicos de vacas primíparas da raça Gir Leiteiro para estimar os componentes de (co)variância das características de conformação, produção de leite em até 305 dias de lactação (PLEI) e idade ao primeiro parto (IPP) pelo Método da Máxima Verossimilhança Restrita (REML). Esses componentes foram utilizados no cálculo dos coeficientes de herdabilidade e nas correlações genéticas, fenotípicas e residuais. As medidas de conformação, circunferência torácica (CTOR), altura na garupa (AG), comprimento corporal (CC), comprimento de tetas (CT), diâmetro de tetas (DT) e altura de úbere (AU) foram realizadas em vacas à primeira lactação, com idade média de 4,05 anos. As correlações genéticas e fenotípicas entre PLEI e IPP foram 0,49 e 0,18, respectivamente. As correlações genéticas entre as características foram: PLEI x CTOR = -0,37; PLEI x AG = -0,62; PLEI x CC = -0,52; PLEI x CT = -0,08; PLEI x DT = -0,12; PLEI x AU = -0,69; IPP x CTOR = -0,05; IPP x AG = -0,37; e IPP x CC = 0,25. As estimativas de correlações genéticas entre produção de leite e medidas de tamanho corporal, de modo geral, indicam que a seleção para produção de leite teria, como resposta correlacionada, diminuição do tamanho da vaca.
\end{abstract}

Palavras-chave: bovino de leite, correlação genética, raça Gir, REML

\section{Estimate of Genetic Parameters of Conformation Traits, Milk Production and Age at First Calving in Dairy Gyr Breed Cows}

\begin{abstract}
Data from 573 primiparous lactating Gyr cows were used to estimate the (co) variance components of the conformation traits, milk production up to 305 days of lactation (MP) and age at first calving (AFC) by REML method. These components were used to calculate heritability coefficients and genetic, phenotypic and residual correlations. The conformation measures, the heart girth (HG), croup height $(\mathrm{CH})$, body length (BL), teats length (TL), teats diameter (TD) and udder height (UH) were obtained in the cows at first lactation with average age of 4.05 years. The genetic and phenotypic correlations between MP and AFC were 0.49 and 0.18 respectively. The genetic correlation between traits were $\mathrm{MP} \times \mathrm{HG}=-0.37$; $\mathrm{MP} \times \mathrm{CH}=-0.62 ; \mathrm{MP} \times \mathrm{BL}=-0.52 ; \mathrm{MP} \times \mathrm{TL}=-0.08 ; \mathrm{MP} \times \mathrm{TD}=-0.12$; $\mathrm{MP} \times \mathrm{UH}=-0.69 ; \mathrm{AFC} \times \mathrm{HG}=-0.05 ; \mathrm{AFC} \times \mathrm{CH}=-0.37$ and $\mathrm{AFC} \times \mathrm{BL}=0.25$. These estimates of genetic correlation between milk production and body size measures, in general, indicate that the selection for milk production held as a correlate response, decreasing in the cow size.
\end{abstract}

Key Words: dairy cattle, genetic correlation, breed Gyr, REML

\section{Introdução}

A participação das raças zebuínas na pecuária brasileira é expressiva, haja vista o número de rebanhos zebus e azebuados explorados para leite, sendo a raça Gir a mais utilizada nos cruzamentos com as raças européias especializadas para produção de leite, na obtenção de mestiços.

A produção de leite, em geral, é a característica mais importante em um programa de melhoramento de gado leiteiro, porém é necessário estudar a sua associação com outras características produtivas, reprodutivas e de conformação, bem como analisar como estas se comportam quando a seleção é praticada para leite.

O "tipo funcional", termo sugerido para se referir à conformação do corpo associada à produção de leite durante a vida produtiva da vaca, foi investigado em vários trabalhos; a maioria dos autores concluíram que as duas características, tipo e produção, parecem ser independentemente herdadas e, para melhorá-las, é necessário que a seleção seja praticada em ambas.

TOUCHBERRY (1951) verificou que correlações entre produção de leite e mensurações de corpo, em

\footnotetext{
${ }^{1}$ Parte da tese apresentada à UFV pelo primeiro autor, como um dos requisitos para obtenção do título de "Magister Scientiae". Financiamento: CNPq.

2 Estudante de doutorado na UFV. E.mail: aaw@alunos.ufv.br; william@alunos.ufv.br

3 Professor do Departamento de Zootecnia da UFV. E.mail: plopes@mail.ufv.br; baja@mail.ufv.br

${ }^{4}$ Pesquisador da EMBRAPA - Gado de Leite - Juiz de Fora - MG. E.mail: rteodoro@cnpgl.embrapa.br; rsverneque@cnpgl.embrapa.br

5 Professor da UFMG. E.mail: martinho@vet.ufmg.br
} 
geral, são pequenas e normalmente não-significativas.

BATRA e McALLISTER (1984) estudaram a relação de algumas medidas de sistema mamário (comprimento e diâmetro de teta e altura de úbere) com produção de leite em vacas Holandesas jovens e verificaram que as correlações genéticas foram pequenas e não-significativas, exceto o diâmetro de teta, que teve correlação negativa com produção de leite.

NORMAN et al. (1988) observaram correlações genéticas negativas e pequenas entre algumas características de tamanho corporal e produção de leite na primeira lactação de vacas Guernsey e Jersey; entretanto, SHORT e LAWLOR (1992) verificaram correlações positivas, porém pequenas, entre características de tamanho e produção de leite em vacas Holandesas.

KLASSEN et al. (1992) estudaram correlações genéticas entre várias características de tipo e características de produção em vacas Holandesas, encontrando correlações baixas entre características de tamanho e tempo de vida produtiva. MISZTAL et al. (1992) investigaram, em vacas primíparas da raça Holandesa, a relação entre produção e características de tipo, sendo observadas pequenas correlações positivas entre características de tamanho e produção e correlações negativas entre produção e características de sistema mamário. Os autores ressaltaram que a seleção somente para a produção de leite pode causar efeitos negativos em algumas características de úbere.

HARRIS et al. (1992), estudando correlações fenotípicas e genéticas entre produção e características de tipo em vacas da raça Guernsey, constataram que a seleção para produção de leite pode causar respostas correlacionadas em características de tipo.

KAWAHARA et al. (1996) estimaram parâmetros genéticos para características produtivas e de tipo em vacas Holandesas e verificaram que a maioria das características de tamanho corporal foram correlacionadas negativamente com produção de leite e longevidade.

Em seus estudos, DUTT e TOMAR (1972) e GUPTA e BHATNAGAR (1979) verificaram que a correlação genética entre produção de leite à primeira lactação e a idade à primeira parição não segue o mesmo sentido, apresentando coeficientes de - $0,54 \mathrm{e}$ $-0,20$, respectivamente, o que mostra que a seleção para diminuir a idade ao primeiro parto poderá melhorar a eficiência produtiva e reprodutiva das vacas. Os resultados reportados por SOLANKI et al. (1976) e RAMOS (1984) foram baixos, 0,13 e 0,12 , respectivamente, e indicaram comportamento fisiológico de independência quase completa da idade à primeira parição, fato também observado por PRASAD e PRASAD (1972).

BHATNAGAR et al. (1983) encontraram correlação genética de 0,77 , indicando associação genética positiva para idade à primeira parição e produção de leite correspondente.

CAMPOS et al. (1994) estimaram parâmetros genéticos para características de produção e reprodução em rebanhos das raças Holandesa e Jersey na Flórida, Estados Unidos, e verificaram que as correlações entre características de produção e reprodução são baixas e geralmente antagônicas.

Os resultados obtidos de estudos para estimar correlações genéticas entre tipo e fertilidade, em bovinos criados em ambiente temperado, indicam que grande parte dessas correlações é próxima de zero.

HANSET et al. (1989) verificaram que as correlações genética e fenotípica entre circunferência e idade ao primeiro parto foram de $-0,55$ e $-0,35$, respectivamente, e aquelas entre altura e idade ao primeiro parto, de $-0,25$ e - 0,22 . Os autores concluíram que a seleção para tamanho corporal torna possível reduzir a idade ao primeiro parto na raça Belgian Blue, naquele sistema de manejo.

WINKLER (1993), estudando a raça Guzerá, verificou que as correlações genéticas entre idade ao primeiro parto e as medidas de tamanho corporal foram levemente positivas (peso, circunferência e altura de cernelha) ou nulas (altura de garupa e comprimento).

Este estudo foi realizado com o objetivo de estimar, por meio da máxima verossimilhança restrita, os parâmetros genéticos e fenotípicos de medidas de conformação, idade ao primeiro parto e produção de leite de vacas da raça Gir Leiteiro.

\section{Material e Métodos}

Neste estudo foram usados os dados de 573 vacas de primeiro parto, da raça Gir Leiteiro, filhas de 107 touros, nascidas no período de 1987 a 1993, mensuradas no período de 1992 a 1996, distribuídas em oito rebanhos, sendo quatro em São Paulo, três em Minas Gerais e um na Paraíba.

Foram avaliadas as características: produção de leite em até 305 dias de lactação (PLEI), idade ao primeiro parto (IPP), circunferência torácica (CTOR), altura de garupa (AG), comprimento corporal (CC), comprimento de teta (CT), diâmetro de teta (DT) e altura de úbere (AU). 
Rev. bras. zootec.

Detalhes de medição das características e do arquivo de dados são descritos por WENCESLAU (1998).

Os componentes de variância e covariância necessários para estimação dos parâmetros genéticos e fenotípicos foram obtidos pelo método da Máxima Verossimilhança Restrita (REML), utilizando-se o sistema MTDFREML ("Multiple Trait Derivative Free Restricted Maximum Likelihood"- BOLDMAN et al., 1995). Na obtenção dos componentes de (co)variância, foi utilizado, para idade ao primeiro parto, um modelo animal incluindo o efeito fixo de grupo contemporâneo (rebanho, ano, estação). Para produção de leite, foi também incluída a covariável idade da vaca ao parto e para as medidas de conformação, o efeito fixo do estádio da lactação e a covariável idade da vaca à mensuração.

Para o cálculo das herdabilidades, as variâncias genética aditiva, residual e fenotípica foram obtidas em análise de características simples. Para o cálculo das correlações genéticas, fenotípicas e residuais as variâncias e covariâncias foram obtidas pela análise das características aos pares (duas a duas).

\section{Resultados e Discussão}

As estimativas dos componentes de variância genética aditiva e residual e das herdabilidades das características de conformação, produção de leite e idade ao primeiro parto são apresentadas na Tabela1 .

A estimativa de herdabilidade de idade ao primeiro parto de 0,56 , observada neste estudo, indica que há variação genético-aditiva suficiente para obtenção de resposta à seleção para menor idade ao primeiro parto.

Sabe-se que os criadores, de modo geral, estabelecem peso mínimo para que as novilhas sejam inseminadas ou cobertas, e as novilhas que atingem esse peso mais precocemente também apresentam o primeiro parto mais cedo. Portanto, estimativas de herdabilidade elevadas de idade ao primeiro parto devem, então, estar relacionadas a altos valores das estimativas de peso corporal.

ODEDRA et al. (1978), MELLO (1994) e SOUZA et al. (1995), todos trabalhando com rebanhos Gir, também encontraram valores elevados de herdabilidade para esta característica em seus estudos. Na raça Guzerá, MIRANDA et al. (1986) e WINKLER (1993) encontraram estimativas de $0,47 \mathrm{e} 0,34$, respectivamente.

Melhorias na alimentação e no manejo das novilhas nas fases de cria e recria reduzem a idade ao primeiro parto.
Tabela 1 - Estimativas de componentes de variância genética aditiva $\left(\hat{\sigma}_{\mathrm{a}}^{2}\right)$ e residual $\left(\hat{\sigma}_{\mathrm{e}}^{2}\right)$ e de herdabilidades $\left(\hat{h}^{2}\right)$, em análises de características simples

Table 1 - Estimates of additive genetic $\left(\hat{\sigma}_{\mathrm{a}}^{2}\right)$ and residual $\left(\hat{\sigma}_{\mathrm{e}}^{2}\right)$ variances and heritabilities $\left(\hat{\mathrm{h}}^{2}\right)$, in single trait analyses

\begin{tabular}{llll}
\hline Característica & $\hat{\sigma}_{\mathrm{a}}^{2}$ & $\hat{\sigma}_{\mathrm{e}}^{2}$ & $\hat{\mathrm{h}}^{2}$
\end{tabular}

Trait

Produção de leite $\quad 158838,5353977400260,87476 \quad 0,28$ Milk production

Idade ao primeiro parto $\quad 23496,5335500 \quad 18344,12378 \quad 0,56$

Age at first calving

Circunferênciatorácica $\quad 9,5561700 \quad 31,75034 \quad 0,23$

Heart girth

Altura de garupa

$4,2753200 \quad 7,29521 \quad 0,37$

Croup height

Comprimento corporal $\quad 2,6761000 \quad 21,14872 \quad 0,11$

Bodylenght

Comprimento de teta $\quad 0,7882400 \quad 0,93137 \quad 0,46$

Teats lenght

Diâmetro de teta

$0,0121300 \quad 0,15696 \quad 0,07$

Teats diameter

Altura de úbere $\quad 3,8693800 \quad 7,32517 \quad 0,35$

Udder height

A estimativa de herdabilidade de produção de leite foi de 0,28 , sendo intermediária às relatadas na literatura, e concorda com os valores encontrados por autores brasileiros que utilizaram dados de rebanhos nacionais da raça Gir. ALBUQUERQUE (1996), utilizando o método da máxima verossimilhança restrita, encontrou o valor estimado de 0,20 em seu estudo com vacas da raça Gir.

A estimativa de herdabilidade de produção de leite encontrada neste estudo indica variabilidade genética dessa população, sendo viável o teste de progênie que vem sendo realizado nessa raça.

A estimativa de herdabilidade de circunferência torácica igual a 0,23 encontrada neste estudo é baixa quando comparada aos resultados relatados por FREEMAN e ROACHE (1961) e WINKLER (1993), que encontraram estimativas de 0,74 e 0,52 em vacas Holandesas e Guzerá, respectivamente, porém concorda com os valores moderados encontrados por HAGGER e HOFER (1991), em vacas das raças Simental e Swiss Braunvieh.

A estimativa da herdabilidade de altura de garupa $(0,37)$ é similar às encontradas por MISZTAL et al. (1992), SMOTHERS et al. (1993) e KAWAHARA et al. (1996), em vacas Holandesas, porém é inferior ao valor de 0,69 encontrado por WINKLER (1993), em vacas Guzerá, e de 0,53, encontrado por HARRIS et al. (1992), em vacas da raça Guernsey. FREEMAN 
e ROACHE (1961) e NAMJOSHI e KATPATAL (1983) também encontraram valores de herdabilidade mais elevados para essa característica.

A estimativa de herdabilidade para comprimento corporal encontrada foi baixa $(0,11)$, sendo similar à relatada por NAMJOSHI e KATPATAL (1983), em vacas mestiças, mas é inferior aos valores $0,58 \mathrm{e}$ 0,41, encontrados por TOUCHBERRY (1951), em vacas Holandesas, e WINKLER (1993), em vacas Guzerá, respectivamente.

As estimativas de herdabilidades das características de sistema mamário, principalmente comprimento de teta e altura do úbere, encontradas neste estudo são semelhantes às relatadas por HIGGINS et al. (1980), BATRA e McALLISTER (1984) e HARRIS et al. (1992). Contudo, a estimativa de herdabilidade de diâmetro de teta, próxima de zero, é diferente da estimativa de 0,33 , encontrada por BATRA e McALLISTER (1984).

De modo geral, as moderadas estimativas de herdabilidades das características de conformação encontradas neste trabalho, associadas à razoável variabilidade genética na população estudada, indicam que a seleção para essas características pode resultar em progresso genético.

$\mathrm{Na}$ Tabela 2, estão as estimativas das correlações genética, fenotípica e residual entre produção de leite e idade ao primeiro parto.

A estimativa de correlação genética entre produção de leite e idade ao primeiro parto foi moderadamente alta e positiva, diferente dos resultados de DUTT e TOMAR (1972) e GUPTA e BHATNAGAR (1979), que verificaram correlações genéticas negativas. Entretanto, os resultados encontrados neste estudo são semelhantes aos observados por BHATNAGAR et al. (1983) e CAMPOS

Tabela 2 - Estimativas de correlações genética $\left(\hat{\mathrm{r}}_{\mathrm{g}}\right)$, fenotípica $\left(\hat{\mathrm{r}}_{\mathrm{P}}\right)$ e residual $\left(\hat{\mathrm{r}}_{\mathrm{e}}\right)$ entre produção de leite (PLEI) e idade ao primeiro parto (IPP)

Table 2 - Estimates of genetic $\left(\hat{\mathrm{r}}_{\mathrm{g}}\right)$, phenotypic $\left(\hat{\mathrm{r}}_{\mathrm{P}}\right)$ and residual $\left(\hat{\mathrm{r}}_{\mathrm{e}}\right)$ correlations between milk production $(M P)$ and age at first calving (AFC)

\begin{tabular}{lcc}
\hline Item & PLEI(MP) & IPP(AFC) \\
\hline Produção de leite (PLEI) & - & 0,49 \\
$\begin{array}{l}\text { Milk production }(M P) \\
\text { Idade ao primeiro parto (IPP) }\end{array}$ & 0,$18 ;(-0,08)$ & - \\
Age at first calving (AFC) & & \\
\hline
\end{tabular}

$\hat{\mathrm{r}}_{\mathrm{g}}$ acima da diagonal; e $\hat{\mathrm{r}}_{\mathrm{P}},\left(\hat{\mathrm{r}}_{\mathrm{e}}\right)$ abaixo da diagonal.

$\hat{\mathrm{r}}_{\mathrm{g}}$ above diagonal; and $\hat{\mathrm{r}}_{\mathrm{p}}$, $\left(\hat{\mathrm{r}}_{\mathrm{e}}\right)$ below diagonal. et al. (1994), indicando que há certa ação gênica comum para idade à primeira parição e produção de leite, sendo esta associação genética antagônica do ponto de vista zootécnico, já que a seleção para as características de produção teriam, como resposta correlacionada, aumento da idade ao primeiro parto.

As estimativas de correlações genéticas, fenotípicas e residuais entre produção de leite e características de conformação, analisadas duas a duas, são apresentadas na Tabela 3 .

As estimativas das correlações genéticas entre produção de leite e características de tamanho corporal foram negativas e moderadamente altas.

Os resultados encontrados neste estudo são diferentes dos obtidos por TOUCHEBERRY (1951), JOHANSSON (1964) e HAGGER e HOFER (1991), que verificaram correlações genéticas baixas e nãosignificativas entre produção de leite e mensurações de corpo. Entretanto, valores de correlações genéticas negativas, porém menores, também foram encontrados por outros autores, como SYRSTAD (1966), MORRIS e WILTON (1976), NORMAN et al. (1988) e KAWAHARA et al. (1996).

As correlações fenotípicas entre produção de leite e características de tamanho corporal foram próximas de zero. Resultados similares foram encontrados por VIJ et al. (1990). As estimativas das correlações residuais foram pequenas e positivas.

As estimativas de correlações genéticas entre produção de leite e comprimento e diâmetro de teta

Tabela 3 - Estimativas de correlações genéticas $\left(\hat{\mathrm{r}}_{\mathrm{g}}\right)$, fenotípicas $\left(\hat{\mathrm{r}}_{\mathrm{P}}\right)$ e residuais $\left(\hat{\mathrm{r}}_{\mathrm{e}}\right)$ entre produção de leite e características de conformação (circunferência torácica = CTOR; altura de garupa $=A G$; comprimento corporal = CC; comprimento de teta $=\mathrm{CT}$; diâmetro de teta $=\mathrm{DT}$; e altura de úbere $=A U)$, analisadas duas a duas

Table 3 - Estimates of genetic $\left(\hat{\mathrm{r}}_{\mathrm{g}}\right)$, phenotypic $\left(\hat{\mathrm{r}}_{\mathrm{P}}\right)$ and residual $\left(\hat{\mathrm{r}}_{\mathrm{e}}\right)$ correlations for milk production (MP), heart girth $(H G)$, croup height $(C H)$, body length $(B L)$, teats length $(T L)$, teats diameter $(T D)$ and udder heigth $(U H)$, in multiple trait analyses (two by two)

\begin{tabular}{|c|c|c|c|c|c|c|}
\hline $\begin{array}{l}\text { Característica } \\
\text { Trait } \\
\text { Produção de leite } \\
\text { Milk production }\end{array}$ & $\begin{array}{c}\text { CTOR } \\
H G\end{array}$ & $\begin{array}{l}\mathrm{AG} \\
\mathrm{CH}\end{array}$ & $\begin{array}{l}\mathrm{CC} \\
B L\end{array}$ & $\begin{array}{l}\text { CT } \\
T L\end{array}$ & $\begin{array}{l}\text { DT } \\
T D\end{array}$ & $\begin{array}{l}\mathrm{AU} \\
U H\end{array}$ \\
\hline$\hat{\mathrm{r}}_{\mathrm{g}}$ & $-0,37$ & $-0,62$ & $-0,52$ & $-0,08$ & $-0,12$ & $-0,69$ \\
\hline$\hat{\mathrm{r}}_{\mathrm{P}}$ & 0,00 & 0,02 & $-0,02$ & 0,06 & 0,16 & $-0,25$ \\
\hline$\hat{\mathrm{r}}_{\mathrm{e}}$ & 0,12 & 0,28 & 0,09 & 0,13 & 0,22 & $-0,02$ \\
\hline
\end{tabular}


foram levemente negativas. Resultados semelhantes foram relatados por BATRA e McALLISTER (1984). Observou-se correlação moderadamente alta e negativa entre produção de leite e distância do úbere ao chão, indicando que a seleção para a produção de leite teria como resposta correlacionada úberes mais baixos; fato também observado por BAKKEN (1981) e MISZTAL et al. (1992), os quais verificaram em vacas Holandesas que a seleção somente para a produção de leite pode causar efeitos negativos em características de úbere.

De modo geral, as correlações genéticas indicam que a seleção para produção de leite teria como resposta correlacionada a diminuição do tamanho geral da vaca. Entretanto, em razão do número de informações deste estudo, as correlações genéticas estimadas podem estar associadas a elevados erros-padrão; portanto, estudos adicionais devem ser realizados para investigar estas associações e relações, já que não existem informações a esse respeito na raça Gir.

As estimativas de correlações genéticas, fenotípicas e residuais entre idade ao primeiro parto e características de tamanho corporal, analisadas duas a duas, são apresentadas na Tabela 4.

Pelos resultados obtidos, verifica-se que as estimativas de correlações genéticas foram de moderadas a baixas e negativas, exceto para a estimativa da correlação entre idade ao primeiro parto e comprimento corporal, que foi levemente positiva. HANSET et al. (1989) encontraram correlações negativas significativas entre medidas de tamanho corporal e

Tabela 4 - Estimativas de correlações genéticas $\left(\hat{\mathrm{r}}_{\mathrm{g}}\right)$, fenotípicas $\left(\hat{\mathrm{r}}_{\mathrm{p}}\right)$ e residuais $\left(\hat{\mathrm{r}}_{\mathrm{e}}\right)$ entre idade ao primeiro parto e características de tamanho corporal (circunferência torácica $=$ CTOR; altura de garupa $=$ AG; comprimento corporal $=\mathrm{CC}$ )

Table 4 - Estimates of genetic $\left(\hat{\mathrm{r}}_{\mathrm{g}}\right)$, phenotypic $\left(\hat{\mathrm{r}}_{\mathrm{p}}\right)$ and residual $\left(\hat{\mathrm{r}}_{\mathrm{e}}\right)$ correlations for age at first calving (AFC), heart girth $(H G)$, croup height $(C H)$, body length $(B L)$, in multiple trait analyses (two by two)

\begin{tabular}{lccc}
\hline Característica & CTOR & AG & CC \\
Trait & $H G$ & $C H$ & $B L$ \\
\hline $\begin{array}{l}\text { Idade ao primeiro parto } \\
\text { Age at first calving }\end{array}$ & & & \\
$\hat{\mathrm{r}}_{\mathrm{g}}$ & $-0,05$ & $-0,37$ & 0,25 \\
$\hat{\mathrm{r}}_{\mathrm{P}}$ & 0,04 & $-0,07$ & 0,10 \\
$\hat{\mathrm{r}}_{\mathrm{e}}$ & 0,10 & 0,21 & 0,05 \\
\hline
\end{tabular}

idade ao primeiro parto e concluíram que a seleção para tamanho pode ter como resposta correlacionada idades mais precoces de parto.

As estimativas das correlações fenotípicas encontradas neste trabalho foram baixas, próximas de zero, e as correlações residuais, baixas e positivas.

As associações entre tamanho corporal e idade ao primeiro parto não foram consistentes o suficiente para se concluir que a seleção para tamanho pudesse reduzir a idade à primeira parição. De acordo com MACKINNON et al. (1990), de maneira geral, as características tamanho corporal e fertilidade têm suas expressões independentes. Pode-se inferir que na raça Gir essas associações seguem a mesma tendência.

\section{Conclusões}

As estimativas de herdabilidades de produção de leite, idade ao primeiro parto e das medidas de conformação, associadas à variabilidade genética encontrada na população estudada, indicam que a seleção para estas características pode resultar em progresso genético.

As estimativas de correlações genéticas entre produção de leite e medidas de tamanho corporal, encontradas neste estudo, permitem verificar que a seleção para produção de leite pode ter, como resposta correlacionada, diminuição do tamanho da vaca.

\section{Agradecimento}

À Universidade Federal de Viçosa (UFV), em especial, ao Departamento de Zootecnia (DZO), pela oportunidade de realização do curso de Mestrado.

À Empresa Brasileira de Pesquisa Agropecuária (Embrapa)/Centro Nacional de Pesquisa de Gado de Leite (CNPGL), e à Associação Brasileira de Criadores de Gir Leiteiro (ABCGIL), pela cessão dos dados do Programa Nacional de Melhoramento Genético da Raça Gir.

Ao Conselho Nacional de Desenvolvimento Científico e Tecnológico (CNPq), pela concessão da bolsa de estudo.

\section{Referências Bibliográficas}

ALBUQUERQUE, M.S.M. Estimativas de parâmetros genéticos e fenotípicos das características de produção nas três primeiras lactações em rebanhos da raça Gir. Jaboticabal, SP: UNESP, 1996, 68p. Tese (Mestrado em Melhoramento Animal) - Universidade do Estado de São Paulo, 1996.

BAKKEN, G. 1981. Relationship between udder and teat 
morphology, mastitis and milk production in Norwegian Red cattle. Acta Agric. Scand., 31:438-444.

BATRA, T.R., McALLISTER, A.J. 1984. Relationships among udder measurements, milking speed, milk yield, and CMT scores in young dairy cows. Can. J. Anim. Sci., 64:807-815.

BHATNAGAR, D.S., TANEJA, V.K., BASU, S.B. et al. 1983. Genetic parameters for some economic traits in Sahiwal cattle. Ind. J. Dairy Sci., 36(4):402-406.

BOLDMAN, K.G., KRIESE, L.A., VAN VLECK, L.D. et al. 1995. A manual for use of MTDFREML. A set of programs to obtain estimative of variances and covariances [DRAFT]. Lincoln: USDA/ARS. 120p.

CAMPOS, M.S., WILCOX, C.J., BECERRIL, C.M. et al. 1994. Genetic parameters for yield and reproductive traits of Holstein and Jersey cattle in Florida. J. Dairy Sci., 77(3):867-873.

DUTT, M., TOMAR, S.P.S. 1972. Effect of nongenetic factors on the inheritance of age at first calving in Hariana cattle. Ind. J. Anim. Sci., 42(5):333-336.

FREEMAN, A.E., ROACHE, K.L. 1961. Body measurements and their use in predicting milk production. J. Dairy Sci., 44:1197.

GUPTA, S.C., BHATANAGAR, D.S. 1979. A note on genetic association among different productive and reproductive traits in Tharparkar cows. Ind. J. Anim. Sci., 49(5):383-385.

HAGGER, C., HOFER, A. 1991. Phenotypic and genetic relationships between wither height, heart girth and milk yield in the Swiss Braunvieh and Simmental breeds. Lvstck. Prod. Sci., 28(3):265-271.

HANSET, R., MICHAUX, C., DETAL, G. 1989. Genetic analysis of some maternal reproductive traits in the Belgian Blue cattle breed. Lvstck. Prod. Sci., 27(1/2):79-96.

HARRIS, B.L., FREEMAN, A.E., METZGER, E. 1992. Genetic and phenotypic parameters for type and production in Guernsey dairy cows. J. Dairy Sci., 75(4):1147-1153.

HIGGINS, S., MOORE, R.K., KENNEDY, B.W. 1980. Heritabilities of teat conformation traits and their relationships with somatic cell count in Holsteins. Can. J. Anim. Sci., 60:231-239.

JOHANSSON, I. 1964. The relation between body size, conformation and milk yield in dairy cattle. Anim. Breed. Abstr., 32:421-435.

KAWAHARA, T., SUZUKI, M., IKEUCHI, Y. 1996. Genetic parameters of production and type traits and longevity in Holstein population. Anim. Sci. and Technology., 67(5):463-475.

KLASSEN, D.J., MONARDES, H.G., JAIRATH, L. et al. 1992. Genetic correlations between lifetime production and linearized type in Canadian Holsteins. J. Dairy Sci., 75(8):2272-2282.

MACKINNON, M.J., HETZEL, D.J.S., COBERT, N.J. et al. 1990. Correlated responses to selection for cow fertility in a tropical beef herd. Anim. Prod., 50(3):417-424.

MELLO, A.A. Resposta à seleção em características de importância econômica em um rebanho da raça Gir. Belo Horizonte, MG: UFMG, 1994. 97p. Tese (Mestrado em Melhoramento Animal) - Universidade Federal de Minas Gerais, 1994.

MIRANDA, J.J.F., PEREIRA, C.S., PEREIRA, J.C.C. et al. Fatores ambientes e genéticos relacionados com a idade à primeira cria e intervalo entre partos no rebanho Guzerá da fazenda Canoas. In: REUNIÃO ANUAL DA SOCIEDADE BRASILEIRA DE ZOOTECNIA, 23, 1986, Campo Grande. Anais... Campo Grande: SBZ, 1986, p.261.
MISZTAL, I., LAWLOR, T.J., SHORT, T.H. et al. 1992. Multiple-trait estimation of variance components of yield traits using an animal model. J. Dairy Sci., 75(2):544-551.

NAMJOSHI, M., KATPATAL, B.G. 1983. Studies on variance and relationship among body surface area, body weight and linear measurements of zebu-taurus crosses. Indian J. Anim. Sci., 53(11):1167-1171.

NORMAN, H.D., POWELL, R.L., WRIGHT, J.R. et al. 1988. Phenotypic and genetic relationship between linear functional type traits and milk yield for five breeds. J. Dairy Sci., $71: 1880$

ODEDRA, B.A., KAUSHIK, S.N., KATPATAL, B.G. 1978. Studies on reproductive characteristics of Gir cattle. Ind. J. Anim. Sci., 48(5):371-373.

PRASAD, R.J., PRASAD, R.B. 1972. A study on genetic and phenotypic parameters of some economic characters at Tharparkar cattle. Ind. Vet. J., 49(12):1199-1206.

RAMOS, A.A. Estudo das características reprodutivas e produtivas de zebuínos leiteiros da raça Gir nos trópicos. Botucatu, SP: UNESP, 1984. 224p. Tese (Livre Docência) Universidade do Estado de São Paulo, 1984.

SHORT, T.H., LAWLOR, T.J. 1992. Genetic parameters of conformation traits, milk yield, and herd lifes Holsteins. $J$. Dairy Sci., 75(7):1987-1998.

SMOTHERS, C.D., PEARSON, R.E., HOESCHELE, I. et al. 1993. Herd final score and its relationship to genetic and environmental parameters of conformation traits of United States Holsteins. J. Dairy Sci., 76(6):1671-1677.

SOLANKI, J.V., PATEL, M.M., SHUKLA, P.K. 1976. Study on genetic and phenotipic correlation between some economically important traits of Gir cattle. Anim. Breed. Abs., 44:580.

SOUZA, E.M., MILAGRES, J.C., SILVA, M.A. et al. 1995. Influências genéticas e de meio ambiente sobre a idade ao primeiro parto em rebanhos de Gir leiteiro. R. Soc. Bras. Zootec., 24(6):926-935.

SYRSTAD, O. Studies on dairy herd records. IV. 1966. Estimates of phenotypic and genetic parameters. Acta Agric. Scand., 16:79-96.

TOUCHBERRY, R.M. 1951. Genetic correlations between five body measurements, weight, type and production in the same individual among Holstein cows. J. Dairy Sci., 34(3):242-55.

VIJ, P.K., BALAIN, D.S., GEORGE, M. et al. 1990. Linear type traits and their influence on milk production in Tharparkar cattle. Indian J. Anim. Sci., 60(7):845-852.

WENCESLAU, A.A. Estudo de medidas de conformação e suas relações com características produtivas e reprodutivas em vacas da raça Gir Leiteiro. Viçosa, MG: UFV, 1998. 75p. Tese (Mestrado em Melhoramento Animal) - Universidade Federal de Viçosa, 1998.

WINKLER, R. Tamanho corporal e suas relações com algumas características reprodutivas em fêmeas bovinas adultas da raça Guzerá. Belo Horizonte, MG: UFMG, 1993. 116p. Tese (Mestrado em Melhoramento Animal) - Universidade Federal de Minas Gerais, 1993.

Recebido em: 30/04/98 Aceito em: 07/10/99 